\title{
A Comparison of the Rheologic Properties of Neonatal and Adult Blood
}

\author{
M. W. RAMPLING, P. WHITTINGSTALL, G. MARTIN, S. BIGNALL, R. P. A. RIVERS, \\ T. J. LISSAUER, AND P. C. BAILEY \\ Departments of Physiology and Biophysics [M.W.R., P.W., G.M.], and Paediatrics [S.B., R.P.A.R., T.J.L., \\ P.C.B.], St. Mary's Hospital Medical School, London W2 IPG UK
}

\begin{abstract}
A number of studies have indicated that the rheologic properties of neonatal blood are different from those of the adult. The frequent administration of blood components to the neonate during intensive care make it important that these differences be established and their causes understood. The purpose of this study was to make a detailed comparison of the rheologic properties of neonatal and adult blood, with particular emphasis on low shear rate viscosity and rouleaux-related phenomena. The viscometric data was obtained from seven preterm (PT) and 18 normal term (NT) babies and compared with those from 18 adults (A). In the present study, viscometry was performed over a wide range of shear rates, from about 0.3 to $130 \mathrm{~s}^{-1}$, and the low shear rate data were compared with direct measurement of rouleaux formation using the Myrenne Erythrocyte Aggregometer. A major factor leading to the viscometric differences observed was the high hematocrit common in the newborn $(46.8 \pm 2.1 \%$ PT, $52.8 \pm$ $6.1 \%$ NT, $44.1 \pm 2.5 \%$ A males, $40.5 \pm 1.9 \%$ A females). However, this tended to be compensated for by the lower plasma viscosity $(1.05 \pm 0.07 \mathrm{mPas} P T, 1.23 \pm 0.14 \mathrm{mPas}$ NT, $1.34 \pm 0.08 \mathrm{mPas} A-$ no sex difference) and reduced rouleaux formation observed in the newborn and more marked in the preterm baby. The lowered levels of red cell aggregation were found not to be due to cellular differences between the adults and the babies but rather to differing plasma components. The presence of the fetal variant of fibrinogen and low levels of immunoglobulins, especially IgM and IgA, are likely to be of particular importance. (Pediatr Res 25:457-460, 1989)
\end{abstract}

A number of studies have shown that the viscometric properties of blood from the neonate are generally different from those of the adult (1-4). In part, this is due to the higher hematocrit common in the newborn (1) and the lower plasma viscosity $(2$, 3 ). Furthermore, it has been reported that rouleaux formation is also reduced in the newborn (5), and this should lead to reduced low shear rate viscosity (6). However, viscometric studies to date have been done at too high a shear rate for rouleaux effects to be obvious, and little information is available to explain the reduced rouleaux formation in the blood of the newborn. The purpose of this study was to make a detailed comparison of the rheologic properties of neonatal and adult blood, with particular

Received October 12, 1987; accepted December 8, 1988.

Address for correspondence Dr. M. W. Rampling, Department of Physiology and Biophysics, St. Mary's Hospital Medical School, London W2 1PG, UK.

Funded by a grant from the National Fund for Research into Crippling Diseases (Action Research for the Crippled Child). S.B. was supported by the St Mary's Hospital Save the Baby Fund and P.C.B. by the North Thames Regional Health Authority. emphasis on low shear rate viscosity and rouleaux-related phenomena.

\section{MATERIALS AND METHODS}

Blood. Blood was obtained from healthy adults by venesection, and from the umbilical vein of neonates immediately after clamping of the cord at delivery. In each case, it was anticoagulated with $12.5 \mathrm{U} / \mathrm{mL}$ of heparin. The babies were all born vaginally and did not require resuscitation. They were of normal wt for gestation age. There were too few preterm babies to group them according to gestational age, which ranged from 24 to 35 wk, thus all babies of less than 37 wk were classified as preterm. The adults had an age range of 24 to $50 \mathrm{y}$ with a median age of 30 .

In a pilot survey, the rheologically important variables-hematocrit, plasma viscosity, and plasma fibrinogen concentrationwere compared between pre- and normal term babies and adults This was followed by a more comprehensive hemorheologic study that included the measurement of blood viscosity over a wide range of shear rates. These viscometric studies were made according to the ICSH Guidelines (7) on blood at native hematocrit and, after adjustment by adding or subtracting autologous plasma, at $45 \%$. The hematocrit was measured by microcentrifugation without correction for trapped plasma.

In some experiments, washed cells were used. The cells were washed, by alternate centrifugation and aspiration of supernatant, using isotonic PBS. The cells were finally suspended at a hematocrit of $45 \%$ in PBS or PBS containing adult human fibrinogen (Kabi Pharmaceuticals, Stockholm, Sweden).

Fibrinogen concentration. Fibrinogen concentration was measured using a thrombin clotting technique (8).

Viscosity measurements. Viscosity measurements were made using a Contraves LS30 Viscometer (Contraves A G, Zurich, Switzerland) with a $1+1$ bob-in-cup system. In all cases, the viscosity of the cell suspension was measured first, then the suspending phase was separated by centrifugation and its viscosity measured on the same instrument.

Index of aggregation. An erythrocyte aggregometer (Myrenne $\mathrm{GmbH}$, Roetgen, West Germany) was used to provide an index of aggregation (9). This is based on a transparent cone-on-plate viscometer head through which a light beam passes. The sample in the gap is subjected to high shear to disrupt any cellular aggregates present. It is then subjected to 0 ( $\mathrm{M}$ mode) or low

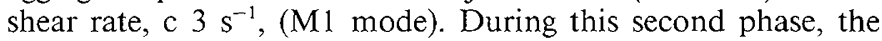
cells aggregate, and the change in light transmission through the sample is integrated over $10 \mathrm{~s}$. This gives a numerical index of aggregation. All samples used on this instrument were adjusted to a $45 \%$ hematocrit.

Statistical comparisons. Comparisons were made using Student's $t$ test. 


\section{RESULTS}

Hematocrit, plasma viscosity, and fibrinogen. The most important determinants of blood viscosity are hematocrit, plasma viscosity and plasma fibrinogen concentration, hence a survey was made of these. The adult hematocrit data were divided as a function of sex; none of the other data showed any sex differences, so these were combined. They are shown in Table 1 . The hematocrits of the two baby groups were not significantly different, but both were different from the adult groups. The plasma viscosities were all significantly different, increasing from preterm to term babies to adults. The fibrinogen concentrations increased in a similar fashion, but only the adult level differed significantly from the other two.

Whole blood viscosity. Full scans of blood viscosity at native hematocrit were performed over a shear rate range from 0.277 $\mathrm{s}^{-1}$ to $128.5 \mathrm{~s}^{-1}$ on several subjects chosen at random. The mean data for each group are plotted in Figure 1. A statistical comparison was also made between the viscosity data of both baby groups and a broader adult group at the extreme shear rates. The numerical data used are shown in Table 2.

The hematocrits of all the groups were significantly different as were the low shear rate viscosities. At high shear rate, the situation was different in that the viscosities of the preterm babies and female adults were similar; otherwise, the same pattern persisted.

Hematocrit-corrected blood viscosity. To remove the effects of hematocrit differences between the groups, the samples were adjusted to a hematocrit of $45 \%$ and the viscosity redetermined. This had little effect on the shape of the viscosity curves shown in Figure 1, but it did move them relative to one another. In particular, the adult viscosity values, at all shear rates, now became significantly higher than those of the term babies, and these higher than the preterm data. This is illustrated in Table 3, where viscosity values at the extreme shear rates are shown. No significant differences were found between the adult male and female data (after hematocrit adjustment) and so these were combined in Table 3 .

Relative viscosity. Plasma viscosity has a substantial role to play in influencing blood viscosity (10), and, as shown in Table 1 , significant differences in plasma viscosity exist between the groups under study. This effect of plasma viscosity can be largely removed by calculating the relative viscosity (11) given by:

\section{blood viscosity at $45 \%$ hematocrit/plasma viscosity}

Effectively, this term gives, at the same hematocrit for each group, the viscosity characteristics of the cellular compartment alone.

This manipulation was performed on the data of Table 3 , and the results are shown in Table 4. There are now no differences between any of the groups at high shear rate. However, at low shear rate, the differences persist.

Low shear rate viscosity and fibrinogen. The differences in low shear rate viscosity between the groups shown in Tables 2-4 even after hematocrit and plasma viscosity correction, are strongly indicative of different levels of rouleaux formation between the groups (12). The major factor in adult blood which leads to rouleaux formation is fibrinogen (6); hence the low shear rate data of the groups were plotted against fibrinogen as shown in Figure 2. The preterm group was not included as the data points were too few, and too closely packed, to allow a trend to be observed. Linear regression analysis was applied to the data, and the correlation coefficients are shown in the legend to Figure 2.

The adult viscosity data show a significant positive correlation with fibrinogen concentration, but the neonatal data do not. This indicates a stronger response to fibrinogen in the adults. Further, it can be seen that at any given fibrinogen concentration the adults exhibit a higher relative viscosity.

Myrenne erythrocyte aggregometer data and fibrinogen. The indices of aggregation produced by this instrument were measured in the adult and neonatal groups and compared with their fibrinogen concentrations. The data are plotted in figure 3. Again there were too few data points for the preterm infants to be included. Linear regression analysis was performed on the data and the correlation coefficients are shown in the legend to Figure 3.

The adult $\mathrm{M}$ mode data show a significant positive correlation with fibrinogen; the neonatal data do not. Both sets of data shown a positive correlation in the M1 mode, but even here the adult data vary significantly more with fibrinogen than those from the neonates. This again indicates that there is a stronger response to fibrinogen in the adult. Also, it can be seen in Figure 3 that at any fibrinogen level the adult reading in both the $\mathrm{M}$ or Ml modes is greater than that of the babies.

Washed cell studies. To see if cellular differences might account, at least in part, for the differences seen in Table 4 and Figures 2 and 3, studies were made of washed cells suspended in PBS to which were added increasing amounts of adult fibrinogen. The only differences in these preparations, therefore, were the concentration of fibrinogen and the presence of adult or neonatal cells. In each case, low shear rate viscosity was measured at $45 \%$ hematocrit, then the supernatant viscosity was measured. The

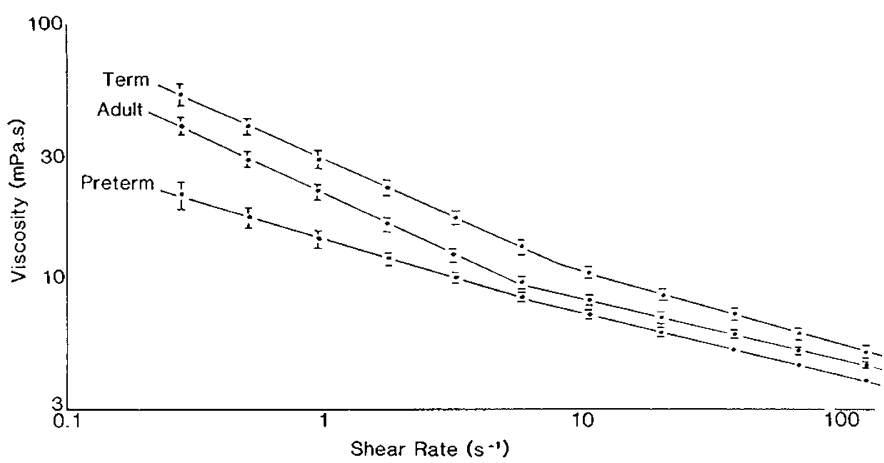

Fig. 1. Whole blood viscosity vs. shear rate plotted on log:log axes. The data represent mean \pm SEM for seven preterm, 18 term infants, and nine adult males. The mean hematocrits $( \pm \mathrm{SD})$ for each group were $46.8 \pm 2.1,52.8 \pm 6.1$, and $44.1 \pm 2.5 \%$, respectively.

Table 1. Hematocrit, plasma viscosity and fibrinogen concentration [mean $\pm S D(n)]$

\begin{tabular}{|c|c|c|c|c|}
\hline & \multicolumn{2}{|c|}{ Neonates } & \multicolumn{2}{|c|}{ Adults } \\
\hline & Preterm & Normal term & Male & Female \\
\hline Hematocrit $(\%)^{*}$ & $48.1 \pm 7.9(14)$ & $49.9 \pm 6.4(60)$ & $44.1 \pm 2.5(19)$ & $40.5 \pm 1.9(6)$ \\
\hline Plasma viscosity $(\mathrm{mPas}) \dagger$ & $1.05 \pm 0.07(6)$ & $1.24 \pm 0.13(22)$ & 1.34 & $\begin{array}{l}\text { (19) } \\
\text { Sexes } \\
\text { combined }\end{array}$ \\
\hline Fibrinogen concentration (g/liter) $\ddagger$ & $2.73 \pm 0.77(14)$ & $2.87 \pm 0.52(60)$ & 3.25 & (17) \\
\hline
\end{tabular}

* All group significantly different $p<0.01$, except pre- and normal term.

$\uparrow$ All groups significantly different $p<0.01$.

$\$$ Neonate groups not different, but differ from adults $p<0.05$. 
Table 2. Hemorheologic data [mean $\pm S D(n)]$ : whole blood viscosity and associated hematocrits

\begin{tabular}{|c|c|c|c|c|c|}
\hline & & \multicolumn{2}{|c|}{ Neonates } & \multicolumn{2}{|c|}{ Adults } \\
\hline & & Preterm & Normal term & Male & Female \\
\hline Hematocrit $(\%)^{*}$ & & $46.8 \pm 2.1(7)$ & $52.8 \pm 6.1(18)$ & $44.1 \pm 2.5(19)$ & $40.5 \pm 1.9(6)$ \\
\hline \multirow{2}{*}{$\begin{array}{l}\text { Viscosity (mPas) at shear } \\
\text { rate of }\end{array}$} & $0.277 \mathrm{~s}^{-1 *}$ & $21.2 \pm 6.6(7)$ & $52.1 \pm 20.7(18)$ & $41.9 \pm 7.1(19)$ & $32.6 \pm 9.4(6)$ \\
\hline & $128.5 \mathrm{~s}^{-1} \dagger$ & $4.00 \pm 0.21(7)$ & $5.11 \pm 1.0(18)$ & $4.6 \pm 0.3(19)$ & $4.1 \pm 0.6(6)$ \\
\hline
\end{tabular}

* All groups significantly different $p<0.05$.

$\doteqdot$ All groups significantly different $p<0.05$, except preterm and female.

Table 3. Hemorheologic data [mean $\pm S D(n)]$ Hematocrit corrected (45\%) viscosity

\begin{tabular}{lcccc}
\hline & & Preterms & Normal terms & Adults \\
\hline $\begin{array}{l}\text { Hematocrit corrected viscosity } \\
\text { (mPas) at shear rates of }\end{array}$ & $0.277 \mathrm{~s}^{-1 *}$ & $18.4 \pm 7.1(7)$ & $30.4 \pm 6.9(18)$ & $44.6 \pm 5.5(22)$ \\
& $128.5 \mathrm{~s}^{-1 *}$ & $3.8 \pm 0.3(7)$ & $4.3 \pm 0.3(18)$ & $4.7 \pm 0.3(22)$ \\
\hline
\end{tabular}

* All groups significantly different $p<0.01$.

Table 4. Hemorheologic data [mean $\pm S D(n)]$ : relative viscosities

\begin{tabular}{lcccc}
\hline $\begin{array}{l}\text { Relative viscosities at shear } \\
\text { rates of }\end{array}$ & $0.277 \mathrm{~s}^{-1 *}$ & $17.3 \pm 5.1(6)$ & $24.3 \pm 3.8(18)$ & $33.2 \pm 3.8(18)$ \\
& $128.5 \mathrm{~s}^{-1}+$ & $3.70 \pm 0.19(6)$ & $3.60 \pm 0.29(18)$ & $3.58 \pm 0.30(18)$ \\
\hline
\end{tabular}

* All groups significantly different $p<0.01$.

$\dagger$ No significant differences.

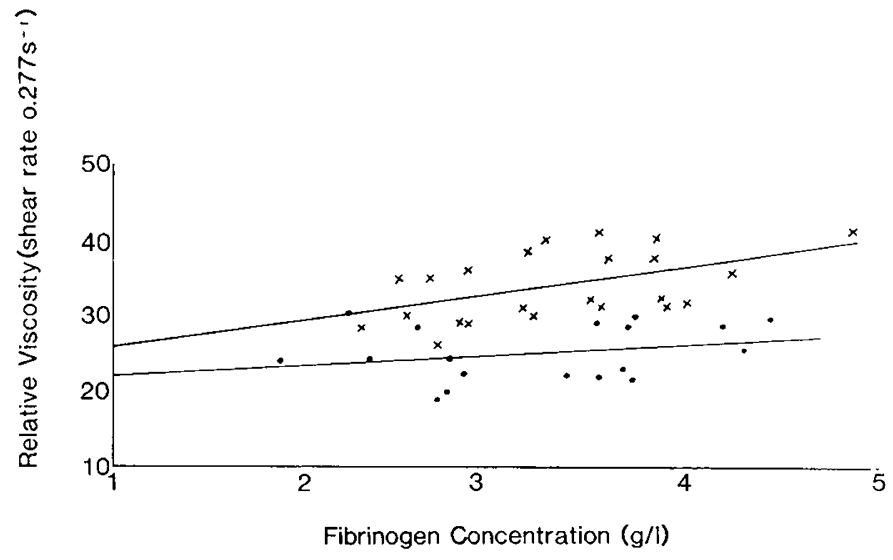

Fig. 2. Relative viscosity at low shear rate $v$ s. plasma fibrinogen for adults $(x)$ and normal term babies $(\bullet)$. The correlation coefficients for the lines are: adults $0.49(p<0.05)$, term babies $0.26(p>0.1)$.

data are plotted as relative viscosity against fibrinogen concentration in Figure 4. There is no discernible difference between the data sets.

\section{DISCUSSION}

It has been known for some time that the rheologic properties of neonatal blood are different from those of the adult and that there is a variation with gestational period (1-4). There are a number of reasons for this: one is the high hematocrit that is common in the neonate (13), another is the lower neonatal plasma protein concentration which leads to a reduced plasma viscosity (3). The mechanical properties of the red cell could also have a role to play, and neonatal cells are generally larger than those of the adult (13). However, erythrocyte deformability is unlikely to be important, as Linderkamp et al. (14) found little difference between adult and neonatal cells of similar vol. A further cellular factor, however, is rouleaux formation, which has been reported to be much lower in neonatal compared with adult blood (5). This should have the effect of lowering blood viscosity

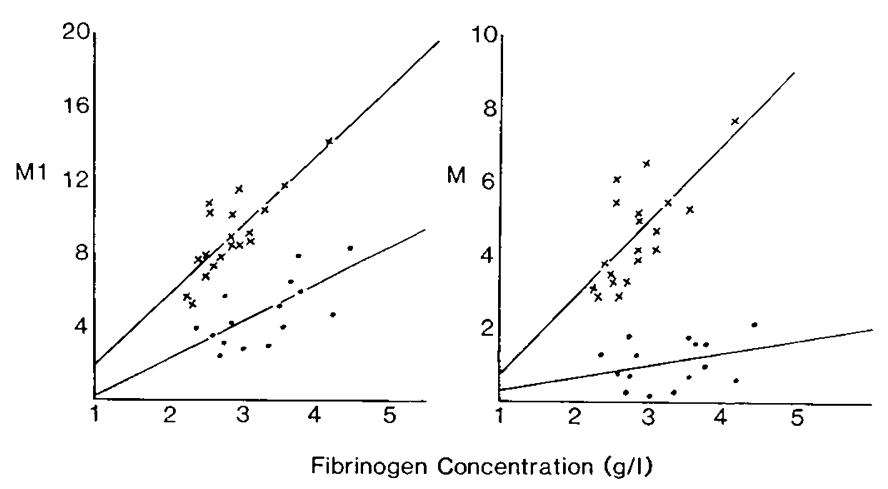

Fig. 3. Myrenne erythrocyte aggregometer reading $v s$. plasma fibrinogen for $(x)$ adults and normal term babies $(\bullet)$. The correlation coefficients for the lines are as follows. Ml data: adults $0.82(p<0.0001)$, term babies $0.67(p<0.01)$; $\mathrm{M}$ data: adults $0.72(p<0.001)$, term babies 0.31 $(p>0.1)$.

at low shear rate (12). In this study, an attempt has been made to elucidate further the causes of the differences between adult and neonatal hemorheology, especially in the relatively unstudied low shear rate region.

The data of this study have shown (Fig. 1 and Tables 2-4) that native blood from the neonate differs viscometrically from that of the adult. In particular, the normal term baby shows higher viscosity at all shear rates. However, it also confirms (Table 1) findings from elsewhere (13) that babies generally have significantly higher hematocrits, which must have rheologic consequences. When the hematocrit effect was removed by making viscosity measurements at a common hematocrit $(45 \%)$, the viscosities of the groups studied were different at all shear rates, increasing now from preterm to term babies to adults.

Part, at least, of the remaining difference must relate to the significant variation in plasma viscosity reported between adults and neonates $(2,3)$, and confirmed here (Table 1$)$. When the effect of this is removed by calculating relative viscosity (Table 4 ), it is found that the differences between the groups disappear in the high shear rate region, i.e. greater than about $6 \mathrm{~s}^{-1}$. This indicates that the high shear rate differences in whole blood 


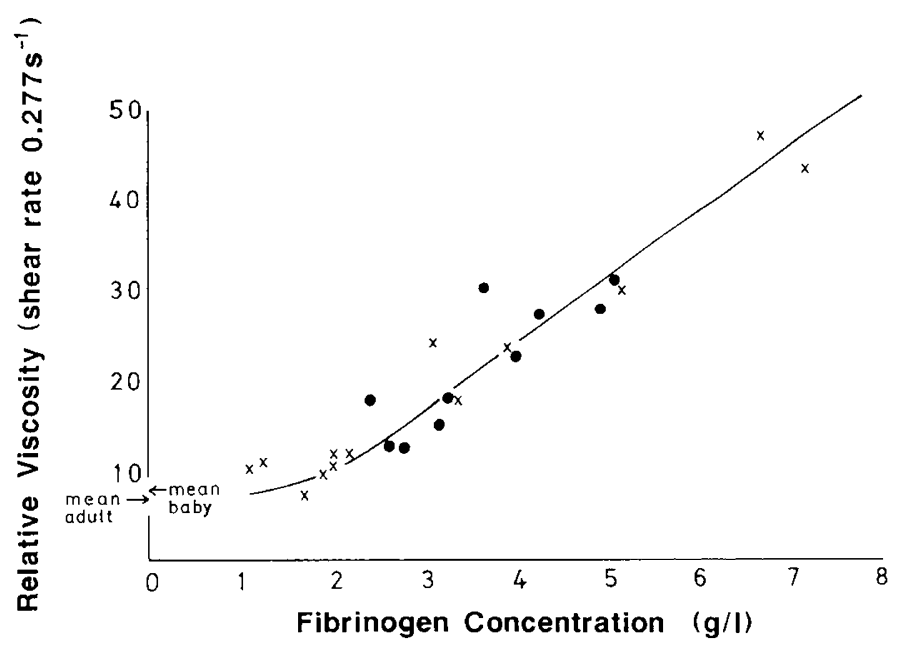

Fig. 4. Relative viscosities of suspensions of washed erythrocytes in PBS containing increasing concentrations of fibrinogen. The neonatal data (-) was obtained from five subjects and the adult $(\times)$ from four.

viscosity between the groups are due solely to variations in hematocrit and plasma viscosity and that cell size and deformability are of little importance. However, at low shear rate (i.e. below $6 \mathrm{~s}^{-1}$ ), differences between the groups still persist and are indicative of differences in the degree of rouleaux formation (12).

It is well known that, in the adult, fibrinogen (6) is the main factor causing rouleaux formation. Thus the correlation with plasma fibrinogen of the indices of aggregation used here, i.e. low shear rate viscosity and the Myrenne erythrocyte aggregometer readings, were studied for each group. These make it clear, as reported elsewhere (5), that neonatal erythrocytes have a lower aggregating tendency than adult cells each in their own plasma. However, the data of Figures 2 and 3 show that this is so even when the fibrinogen levels are the same. Furthermore, these results indicate that babies' cells respond less strongly at a given fibrinogen concentration than do adult cells. Hence variations in fibrinogen concentration between the groups cannot explain the results.

There are several possible reasons for these findings: 1 ) the neonatal cells have inherently reduced rouleaugenic properties; 2 ) alterations in aggregating components in the plasma; 3 ) both of these. To throw light on the first, low shear rate characteristics of washed adult or neonatal cells suspended in PBS with added adult fibrinogen were compared (Fig. 4). No significant differences were observed, and this corresponds to data obtained by Linderkamp et al. (5), who used dextran as the aggregating agent. This suggests that the differing cellular aggregating potential of the groups studied here is due entirely to differences in the protein make-up of the plasma.

It is known that a fetal variant of fibrinogen exists that is characterised by an enhanced sialic acid content by comparison with the adult form (15). Evidence exists suggesting that the charge on the fibrinogen molecule can affect its interaction with the red cell surface (16) and in particular that desialated fibrinogen adsorbs more to the red cell than the native form (17). Thus it is to be expected that the extra, charged sialic acid on fetal fibrinogen will have the reverse effect, i.e. reduce the cellular interaction and hence its rouleaugenic action. However, there is great overlap between the sialic acid contents of adult and fetal fibrinogens (15), so this is unlikely to be the complete answer. The other possibility is low concentration of aggregating proteins apart from fibrinogen. A number of plasma proteins have rouleaugenic capabilities, albeit less than that of fibrinogen itself, e.g. IgM, IgA, IgG, and $\alpha_{2}$-macroglobulin $(18-20)$. What is more, they seem to be able to potentiate the effects of fibrinogen (21). This is probably of relevance here because IgM and IgA levels are extremely low in the newborn (22). Hence the low level of rouleaux formation, and the low viscosity at low shear rate that it brings with it, is probably due to the presence of the fetal variant of fibrinogen and the low concentration of some immunoglobulins. It could also explain the variation of low shear rate relative viscosity with gestational age.

There are a number of fairly common clinical conditions in neonatology where manipulation of blood composition is an accepted form of therapy. These include exchange transfusion for blood group incompatibilities, hemodilution for the hyperviscosity syndrome secondary to polycythemia, and circulatory support in conditions of reduced blood vol. In all of these, adult blood products may be used either as whole blood, fresh frozen plasma, or albumin infusions. The data reported above indicate that disturbing the neonatal plasma environment with adult blood products may have a significant effect on neonatal hemorheology that may not be beneficial. It is important that these effects be investigated and quantitated.

\section{REFERENCES}

1. Bergqvist G 1974 Viscosity of the blood in the newborn infant. Acta Paediatr Scand 63:858-864

2. Linderkamp O, Meiselman HJ, Wu PYK, Miller FC 1981 Blood and plasma viscosity and optimal hematocrit in the normal newborn infant. Clin Haematol 1:575-584

3. Linderkamp O, Versmold HT, Riegel KP, Betke K 1984 Contributions of red cells and plasma to blood viscosity in preterm and full-term infants and adults. Pediatrics 74:645-651

4. Folley ME, Collins R, Macdonald D 1984 Whole blood viscosity in umbilical cord blood, adult pregnant and adult non-pregnant blood: the influence of plasma factors. In: Heilmann L, Buchan PC (eds). Haemorheological Disorders in Obstetrics and Neonatology. Schattauer Verlag, Stuttgart, pp 9297

5. Linderkamp O, Ozanne P, Wu PYK, Meiselman HJ 1984 Red blood cell aggregation in preterm and term neonates and adults. Pediatr Res 18:13561360

6. Rampling MW 1986 The fibrinogen: red cell interaction and its influence on blood rheology. In: Lane DA, Henschen A, Jasani M (eds) Fibrinogen-fibrin Formation and Fibrinolysis, vol 4. Walter de Gruyter, Berlin, pp 219-227

7. International Committee for Standardisation in Haematology 1986 Guidelines for measurement of blood viscosity and erythrocyte deformability. Clin Hemorheol 6:439-453

8. Rampling MW, Gaffney PJ 1976 The sulphite precipitation method for fibrinogen measurement: its use in small samples in the presence of fibrinogen degradation products. Clin Chim Acta 67:43-52

9. Schmid-Schonbein H, Volger E, Teitel P, Kiesewetter H, Dauer V, Heilmann L 1982 New hemorheological techniques for the routine laboratory. Clin Hemorheol 2:93-105

10. Whitmore RL 1968 Rheology of the circulation. Pergamon Press, Oxford, pp 63-89

11. Whitmore RL 1968 Rheology of the circulation. Pergamon Press, Oxford, pp $12-15$

12. Meiselman HJ 1980 Measures of blood rheology and erythrocyte mechanics. In: Cokelet GR, Meiselman HJ, Brooks DE (eds) Erythrocyte Mechanics and Blood Flow. Alan Liss, New York, pp 119-140

13. Oski FA, Naiman JL 1982 Hematologic problems in the newborn. WB Saunders, Philadelphia, pp 1-31

14. Linderkamp O, Nash GB, Wu PYK, Meiselman HJ 1986 Deformability and intrinsic material properties of neonatal red blood cells. Blood 67: 12441250

15. Francis JL, Armstrong DJ 1982 Sialic acid and enzymic desialation of cord blood fibrinogen. Haemostasis 11:223-228

16. Rampling MW 1981 The binding of fibrinogen and fibrinogen degradation products to the erythrocyte membrane and its relationship to haemorheology. Acta Biol Med Germ 40:373-378

17. Rampling MW 1981 Rouleaux formation and fibrinogen related molecules. Proceedings of 2nd European Congress on Clinical Haemorheology. London, p 201

18. Chien S. Usami J, Taylor HM, Lundberg JL, Gregersen MI 1966 Effects of haematocrit and plasma proteins on human blood rheology at low shear rates. J Appl Physiol 21:81-87

19. Schmid-Schonbein H, Gallasch G, Volger E, Klose HJ 1973 Microrheology and protein chemistry of pathological red cell aggregation (blood sludge) studied in vitro. Biorheology 10:213-227

20. Rovel A, Vigneron C, Streiff F 1979 Comparison of in vitro effects of normal $\mathrm{IgG}$ and of monoclonal $\mathrm{IgG}$ on the rheological behaviour of erythrocytes. $\mathrm{Br}$ J Haematol 41:509-513

21. Chien S, Usami J, Dellenback RJ, Gregersen MI 1970 Shear dependent interaction of plasma proteins with erythrocytes in blood rheology. Am J Physiol 219:143-153

22, Nathan DE, Oski FA 1981 Haematology of infancy and childhood. WB Saunders, Philadelphia, pp 1569-1570 